\title{
Singularities in Fuchsian Cauchy Problems with Holomorphic Data
}

\author{
By
}

\author{
Hideshi YAmAnE*
}

\begin{abstract}
It has been proved by Baouend and Goulaouic that the holomorphic Cauchy problem for a Fuchsian partial differential operator of weight $m-m^{\prime}$ has a unique solution if its characteristic exponents avoid integers $\geq m-m^{\prime}$. In the present paper we consider the singularity of the solution which appears if some of the characteristic exponents take integral values $\geq m-m^{\prime}$
\end{abstract}

\section{§1. Introduction}

Let us consider a local Cauchy problem in a neighborhood of the origin $(0,0) \in \mathbb{C}_{t} \times \mathbb{C}_{x}^{n}, x=\left(x_{1}, \ldots, x_{n}\right)$. of the following form.

Let $\Omega$ be an open neighborhood of $0 \in \mathbb{C}_{x}^{n}$ and $P=P\left(t, x, D_{t}, D_{x}\right)$ be a partial differential operator of order $m$ with holomorphic coefficients; here $D_{t}=\partial / \partial t$ and $D_{x}=\left(D_{1}, \ldots, D_{n}\right)=\left(\partial / \partial x_{1}, \ldots, \partial / \partial x_{n}\right)$. We assume that $P$ is a Fuchsian operator of weight $m-m^{\prime}$, that is, it has the form

$$
\begin{aligned}
P\left(t, x, D_{t}, D_{x}\right)= & t^{m^{\prime}} D_{t}^{m}+a_{m-1}(x) t^{m^{\prime}-1} D_{t}^{m-1}+\cdots+a_{m-m^{\prime}}(x) D_{t}^{m-m^{\prime}} \\
& +\sum_{\jmath=0}^{m-1} \sum_{|\beta| \leqq m-j} t^{\alpha(\jmath)} a_{j \beta}(t, x) D_{t}^{\prime} D_{x}^{\beta},
\end{aligned}
$$

where $0 \leq m^{\prime} \leq m, \alpha(j)=\max \left\{0, j-\left(m-m^{\prime}\right)+1\right\}, D_{x}^{\beta}=D_{1}^{\beta_{1}} \cdots D_{n}^{\beta_{n}}$, and $|\beta|=$ $\beta_{1}+\cdots+\beta_{n}$. Here $a_{m-\jmath}(x) \quad\left(j=1, \ldots, m^{\prime}\right)$ and $a_{s \beta}(t, x)(j=0, \ldots, m-1,|\beta|$ $\leq m-j)$ are holomorphic functions in $\Omega$ and $\{t:|t|<T\} \times \Omega, T>0$, respectively.

We call the operator

$$
P_{m}\left(t, x, D_{t}, \widehat{D}_{x}\right)=t^{m^{\prime}} D_{t}^{m}+a_{m-1}(x) t^{m^{\prime}-1} D_{t}^{m-1}+\cdots+a_{m-m^{\prime}}(x) D_{t}^{m-m^{\prime}}
$$

Communicated by T. Kawa1, August 13, 1997. Revised October 22, 1997.

1991 Mathematıcs Subject Classifications: 35A20

* Department of Mathematics, Chiba Instıtute of Technology, Shıbazono, Narashıno 275-0023, Japan.

e-mail. yamane@cc.it-chıba ac.jp 
the Fuchsian principal part of $P$.

The characteristic polynomial associated with $P$ is

$$
\begin{aligned}
C(\lambda, x)= & t^{-\lambda} t^{m-m^{\prime}} P_{m} t^{\lambda} \\
= & \lambda(\lambda-1) \cdots(\lambda-m+1)+a_{m-1}(x) \lambda(\lambda-1) \cdots(\lambda-m+2) \\
& \quad \cdots+a_{m-m^{\prime}}(x) \lambda(\lambda-1) \cdots\left(\lambda-m+m^{\prime}+1\right)
\end{aligned}
$$

and its roots, called the characteristic exponents, are denoted by

$$
\lambda_{1}(x), \ldots, \lambda_{m^{\prime}}(x), \lambda_{m^{\prime}+1}=0, \lambda_{m^{\prime}+2}=1, \ldots, \lambda_{m}=m-m^{\prime}-1 \text {. }
$$

The study of this kind of operators was pioneered by Baouendi and Goulaouic ([2]). They proved the following

Theorem. [Baouendi-Goulaouic] Let $x^{*}$ be a point in $\Omega$. If $C\left(\lambda, x^{*}\right) \neq 0$ for any integer $\lambda \geq m-m^{\prime}$, then for any function $f(t, x)$ holomorphic near $(t, x)=\left(0, x^{*}\right)$ and for any functions $f_{0}(x), \ldots, f_{m-m^{\prime}-1}(x)$ holomorphic near $x=x^{*}$, there exists a unique holomorphic function $u(t, x)$ near $(t, x)=\left(0, x^{*}\right)$ such that

$$
\text { (IVP) }\left\{\begin{array}{l}
P u=f, \\
D_{t}^{\lambda} u(0, x)=f_{\lambda}(x) \quad\left(0 \leq \lambda \leq m-m^{\prime}-1\right)
\end{array}\right.
$$

holds.

In the present paper we consider (IVP) under the following conditions:

- $C(\lambda, 0)=0$ for some integer $\lambda \geq m-m^{\prime}$ and $C(\lambda, x) \neq \equiv$ for any fixed integer $\lambda \geq m-m^{\prime}$,

- $f(t, x)$ is holomorphic near $(t, x)=(0,0)$,

- $f_{0}(x), \ldots, f_{m-m^{\prime}-1}(x)$ are holomorphic near $x=0$.

The set $V$ defined by

$$
V=\left\{x \in \Omega ; C(\lambda, x)=0 \text { for some integer } \lambda \geq m-m^{\prime}\right\}
$$

is an analytic set of codimension 1 of $\Omega$. More precisely, if we define $V_{\lambda}=\{x \in \Omega$; $C(\lambda, x)=0\}$ for a fixed $\lambda \geq m-m^{\prime}$, then $\left\{V_{\lambda}\right\}_{\lambda \geq m-m^{\prime}}$ is a locally finite family of analytic sets of $\Omega$ and we naturally have $V=U_{\lambda \geq m-m^{\prime}} V_{\lambda}$.

For any $x^{*} \in \Omega \backslash V$, the assumption of the above theorem is satisfied and $V$ is an obstruction to the analytic continuation of the solution $u$. A natural question is how far it can be extended near $V$.

Let $d(x)$ be the distance from $x \in \Omega$ to the boundary of $\Omega \backslash V$. If $x$ is in a sufficiently small neighborhood of $x=0 \in \mathbb{C}_{x}^{n}$, then $d(x)$ is nothing but the distance from $x$ to $V$.

We have

Proposition $\mathbb{1}$. The solution $u(x)$ to (IVP) is holomorphic in a domain of the form 


$$
\left\{(t, x) ;|t|<\widetilde{C} d(x)^{m}, x \in \widetilde{\Omega}\right\}
$$

where $\widetilde{\Omega}$ is an open neighborhood of $x=0$ and $\widetilde{C}$ is a positive constant independent of $t$ and $x$.

In fact we can prove a much more precise result. It shall be stated in a formulation involving a quantity $I(P), 0 \leq I(P) \leq m$, which describes the difference between the operator $P$ and its Fuchsian principal part $P_{m}$. It is defined by using something like a Newton polygon.

Recall that

$$
\begin{aligned}
P-P_{m} & =\sum_{j=0}^{m-1} \sum_{|\beta| \leq m-\jmath} t^{\alpha(j)} a_{j \beta}(t, x) D_{t}^{j} D_{x}^{\beta}, \\
\alpha(j) & =\max \left\{0, j-\left(m-m^{\prime}\right)+1\right\} .
\end{aligned}
$$

By expanding $a_{\rho_{\beta}}$ into a power series in $t$, it can be written in the form

$$
\begin{gathered}
P-P_{m}=\sum_{j=0}^{m-1} \sum_{|\beta| \leq n->} \sum_{i=0}^{\infty} t^{2} a_{i j \beta}(x) D_{t}^{j} D_{x}^{\beta} . \\
a_{i j \beta}(x) \equiv 0 \text { if } \quad i \leq j-\left(m-m^{\prime}\right) .
\end{gathered}
$$

For each $j=0,1, \ldots, m-1$, we define a subset $N,(P)$ of $\{1,2, \ldots\} \times\{m-j\}$ by

$$
(k, m-j) \in N_{j}(P) \Longleftrightarrow a_{k+\jmath-\left(m-m^{\prime}\right), \beta, \beta}(x) \not \equiv 0 \text { for some } \beta \text { with } j+|\beta|=m \text {. }
$$

Obviously $N_{j}(P)$ is determined by the principal part of $P$. Let $N(P)$ be the union of $N_{0}(P), N_{1}(P), \ldots$, and $N_{m-1}(P)$. Then we see that $N(P)$ is a subset of $\{1,2, \ldots\} \times(1, \ldots, m\}$.

We set

$$
I(P)=\max \{l / k:(k, l) \in N(P)\} .
$$

If $N(P)=\emptyset$, then we set $I(P)=0$. It is obvious that $0 \leq I(P) \leq m$.

Let $k_{j} \geq 1$ be the smallest $k$ such that $(k, m-j) \in N_{j}(P)$. We set $k_{j}=\infty$ if $N_{j}(P)=\emptyset$ by convention. We have

$$
I(P)=\max _{j} \frac{m-j}{k_{j}} .
$$

Now we can state

Main Theorem. The solution $u(t, x)$ to (IVP) is holomorphic in a domain of the form

$$
\left\{(t, x) ;|t|<\widetilde{C} d(x)^{I(P)}, x \in \widetilde{\Omega}\right\},
$$

where $\widetilde{\Omega}$ is an open neighborhood of $x=0$ and $\widetilde{C}$ is a positive constant independent of $t$ and $x$. If $I(P)=0$, this domain is understood to be 


$$
\{(t, x) ;|t|<\widetilde{C}, \quad x \in \widetilde{\Omega} \backslash V\} .
$$

Some authors have studied Fuchsian Cauchy problems with singular data or with a characteristic exponent which takes a non-generic value: see [4] and [5], for example. In these papers the singularity of the initial data or the set $V$ (in the present author's notation) are assumed to be a nonsingular hypersurface. Moreover they study the cases where the principal symbol of the operators admit some particular types of factorization, which enables the construction of nice phase functions.

On the other hand, in the present paper the set $V$ can be an arbitrary analytic set of codimension 1 and no condition is imposed on the factorization of the principal symbol.

\section{§2. Integral Operators}

In this section we follow [2].

Let $Q\left(t, x, D_{t}, D_{x}\right)$ be a Fuchsian operator of order $m$ and of weight 0 . Denote by $\mu_{r}(x)(r=1,2, \ldots, m)$ its characteristic exponents and assume that Re $\mu_{r}(0)<0$ for all $r$. Then in a sufficiently small neighborhood of the origin we have $\operatorname{Re} \mu_{r}(x)<0$ for all $r$.

If $g(t, x)$ is holomorphic near $(t, x)=(0,0)$, then

$$
\begin{aligned}
v(t, x) & =H[g](t, x) \\
& =\int_{[0,1]^{m}} s_{1}^{-\mu_{1}(3)-1} \cdots s_{m}^{-\mu_{m}(x)-1} g\left(s_{1} \cdots s_{m} t, x\right) d s_{1} \cdots d s_{m}
\end{aligned}
$$

gives the unique holomorphic solution to $Q_{m} v=g$, where $Q_{m}$ is the Fuchsian principal part of $Q$.

We have another representation of $H[g]$ :

$$
H[g]=\frac{1}{m !} \sum_{\pi \in S m} \int_{[0,1]^{m}} s_{\pi(1)}^{-\mu_{1}(1)-1} \cdots s_{\pi(m)}^{-\mu_{m}^{\prime}, 1-1} g\left(s_{1} \cdots s_{m} t . x\right) d s_{1} \cdots d s_{m},
$$

where $S_{m}$ is the group of permutations of $\{1,2, \ldots, m\}$. Its holomorphy is verified by applying the following lemma to $\sum_{\pi \in S m} s_{\pi(1)}^{z_{1}} \cdots s_{\pi(m)}^{z_{m}}$.

Lermma 1 . Let $F(z)$ be an entive function on $\mathbb{C}_{z}^{m}, z=\left(z_{1}, \ldots, z_{m}\right)$, which is symmetric:

$$
F\left(z_{1}, \ldots, z_{m}\right)=F\left(z_{\pi(1)}, \ldots, z_{\pi(m)}\right) \quad \text { for any } \pi \in S_{m} \text {. }
$$

Define $p_{1}(z), \ldots, p_{m}(z)$ by

$$
\begin{aligned}
& \left(X-z_{1}\right)\left(X-z_{2}\right) \cdots\left(X-z_{m}\right) \\
= & X^{m}-p_{1} X^{m-1}+p_{2} X^{m-2}+\cdots+(-1)^{m-1} p_{m-1} X+(-1)^{m} p_{m},
\end{aligned}
$$

where $X$ is an indeterminate. Then there exists a unique entire function $G\left(p_{1}, \ldots, p_{m}\right)$ 
on $\mathbb{C}_{p}^{m}, p=\left(p_{1}, \ldots, p_{m}\right)$, such that $F(z)=G\left(p_{1}(z), \ldots, p_{m}(z)\right)$.

Proof. There exists a sequence of symmetric polynomials $\left\{F_{k}(z)\right\}$ with $\operatorname{deg} F_{k}=k$ such that $F_{k}$ converges to $F$ locally uniformly. We can find a polynomial $G_{k}(p)$ in $p$ of degree $k$ such that $F_{k}(z)=G_{k}\left(p_{1}(z), \ldots, p_{m}(z)\right)$ holds.

On the other hand, for any $R>0$ there exists $N>0$ such that $|p(z)|<R$ implies $|z|<N$.

Therefore $\left\{G_{k}(p)\right\}$ is a Cauchy sequence on $|p|<R$, hence it converges to an entire function $G(p)$.

We see that if $x$ is sufficiently close to the origin there exists a positive constant $C$ independent of $g$ and $(t, x)$ such that

$$
|H[g](t, x)| \leq C \sup _{|\tau| \leq i t \mid}|g(\tau, x)| .
$$

In the proof of Main Theorem, we will need other estimates on $H[g]$. Set, for $k=0,1, \ldots, m$,

$$
H_{k}[g](t, x)=\int_{[0,1]^{k}} s_{1}^{-u_{1}(2)-1} \cdots s_{k}^{-\mu_{k}(x)-1} g\left(s_{1} \cdots s_{k} t, x\right) d s_{1} \cdots d s_{k} .
$$

It is trivial that $H_{m}[g]=H[g]$. For $k<m$ the function $H_{k}[g]$ is not necessarily holomorphic in $x$, but we do have the estimate

$$
\left|H_{k}[g](t, x)\right| \leq C_{k} \sup _{|\tau| \leq|t|}|g(\tau, x)| .
$$

where $C_{k}$ is a positive constant independent of $g$ and $(t, x)$.

By the repeated application of the formula

$$
t D_{t} H_{k}[g]=\mu_{k}(x) H_{k}[g]+H_{k-1}[g] .
$$

we see that, if $x$ is sufficiently close to the origin, we have

$$
\left|\left(t D_{t}\right)^{\prime} H[g](t, x)\right| \leq C^{\prime} \sup _{|\tau| \leq|t|}|g(\tau, x)| \quad(j=0,1 \ldots m) .
$$

where $C^{\prime}$ is a positive constant independent of $g$ and $(t, x)$. Remark that $\left(t D_{t}\right)^{\prime} H$ is a bounded (integral) operator, while $\left(t D_{t}\right)^{\prime}$ itself is not.

Since $\left(D_{t} t\right)^{j}$ is a linear combination of $1, t D_{t} \ldots,\left(t D_{t}\right)^{\prime}$, we obtain the following

Proposition 2. If $x$ is sufficiently close to the origin, there exists a positive constant $A$ independent of $g$ and $(t, x)$ such that

$$
\left|\left(D_{t} t\right)^{\prime} H[g](t, x)\right| \leq A \sup _{|\tau| \leq|t|}|g(\tau, x)| \quad(j=0,1 \ldots m) .
$$

Remark. We prefer $D_{t} t$ to $t D_{t}$ because the former is a topological automorphism on the Fréchet space of holomorphic functions while the latter is neither surjective nor injective. 
We introduce another integral operator which will be useful later. Set

$$
G_{l}[g](t)=\int_{[0,1]^{\prime}} g\left(s_{1} \cdots s_{l} t\right) d s_{1} \cdots d s_{l} \text { for } g=g(t) .
$$

Then it is easy to see that $G_{l}\left(D_{t} t\right)^{l}=i d e n t i t y$ and that $\left(D_{t} t\right)^{j}=G_{m-j}\left(D_{t} t\right)^{m}$. Moreover we have

Lemma 2. If $|g(t)| \leq|t|^{p}$ then we have

$$
\left|G_{l}[g](t)\right| \leq \frac{|t|^{p}}{(p+1)^{l}}
$$

Proof. $\left|G_{l}[g](t)\right| \leq \int_{0}^{1} d s_{1} \cdots \int_{0}^{1} d s_{l}\left\{s_{1}^{p} \cdots s_{l}^{p}|t|^{p}\right\}$

\section{§3. Proof of the Weight 0 Case}

In this section we prove Main Theorem in the case $m=m^{\prime}$; that is, the operator $P$ is assumed to be of weight 0 . In this case the initial condition in (IVP) is null.

First, by writing $f(t, x)=\sum_{\lambda=0}^{\infty} f_{\lambda}(x) t^{\lambda}, u(t, x)=\sum_{\lambda=0}^{\infty} u_{\lambda}(x) t^{\lambda}$, we have the following recurrence relation:

$$
\begin{aligned}
& C(0, x) u_{0}(x)=f_{0}(x) \\
& C(\lambda, x) u_{\lambda}(x)=f_{\lambda}(x)+\sum_{\nu=0}^{\lambda-1} P_{\lambda}^{\nu}\left(x, D_{x}\right) u_{\nu}(x), \lambda=1,2, \ldots,
\end{aligned}
$$

for some differential operator $P_{\lambda}^{\nu}\left(x, D_{x}\right)$. The function $C(\lambda, x)$ may vanish on an analytic set containing the origin, but it is assumed not to vanish identically. Hence each $u_{\lambda}(x)$ is uniquely determinded as a meromorphic function whose singular set is included in $U_{\nu \leq \lambda} V_{\nu} \subset V$.

Next assume that $\operatorname{Re} \lambda_{r}(0)<h \in \mathbb{N}$ for $r=1, \ldots, m$. Obviously $Q=t^{-h} P t^{h}$ is an $m$-th order Fuchsian operator of weight 0 . We denote its characteristic exponents by $\mu_{r}(x)$. Then we have $\mu_{r}(x)=\lambda_{r}(x)-h$ and $\operatorname{Re} \mu_{r}(0)<0$ for all $r$. Moreover we can prove that $N(P)=N(Q)$.

Put $u(t, x)=\sum_{\lambda=0}^{h-1} u_{\lambda}(x) t^{\lambda}+t^{h} v(t, x)$. Then we have

$$
P\left(t^{h} v\right)=f-P\left(\sum_{\lambda=0}^{h-1} u_{\lambda}(x) t^{\lambda}\right)
$$

which is equivalent to the equation

$$
Q v=t^{-h}\left\{f-P\left(\sum_{\lambda=0}^{h-1} u_{\lambda}(x) t^{\lambda}\right)\right\}
$$

The right hand side, which we denote by $g(t, x)$, is holomorphic in $t$ but 
singular in $x$ along $V$.

We have only to solve $Q v=g$ under the following conditions:

- $\operatorname{Re} \mu_{r}(x)<0$ in a neighborhood of $x=0$ for $r=1,2, \ldots, m$,

- there exist a positive constant $C_{g}$ and a positive integer $m_{g}$ such that $|g(t, x)| \leq C_{g} / d(x)^{m_{g}}$ in a neighborhood of $(t, x)=(0,0)$.

Here the estimate on $g$ is a consequence of the following lemma, which is proved in a more precise form in Appendix of [1].

Lemma 3. Let $F(x)$ be a holomorphic function in a neighborhood of the origin of $\mathbf{C}_{x}^{n}$. Assume that $F(0)=0, F \neq 0$. Denote by $d(x)$ the distance from a point $x$ to $\{x ; F(x)=0\}$. Then in a sufficiently small neighborhood of the origin, there exists a positive constant $C$ and a positive integer $M$ such that

$$
|F(x)| \geq C d(x)^{M} .
$$

Put

$$
Q-Q_{m}=\sum_{j=0}^{m-1} \sum_{|\beta| \leq m-j} \sum_{k=0}^{\infty} t^{k} b_{k+j, j, \beta}(x)\left(t^{j} D_{t}^{\jmath}\right) D_{x}^{\beta}
$$

Then $(k, m-j) \in N_{\jmath}(Q)=N_{,}(P)$ if and only if $b_{k+\jmath, j, \beta}(x) \not \equiv 0$ for some $\beta$ with $|\beta|=m-j$. Recall that $k_{j} \geq 1$ is the smallest $k$ such that $(k, m-j) \in N,(Q)=N_{j}(P)$. Then $Q_{m}-Q$ has the following expression:

$$
Q_{m}-Q=\sum_{\jmath=0}^{m-1}\left\{t^{k_{j}} R,\left(t, x, D_{x}\right)+t S_{\jmath}\left(t, x, D_{x}\right)\right\}\left(D_{t} t\right)^{\jmath}
$$

where ord $R_{j}=m-j$ if $N_{j}(Q) \neq \emptyset$ and $R_{j}=0$ if $N_{j}(Q)=\emptyset$. Moreover we have ord $S_{j} \leq m-j-1$ and $\left[R_{j}, t\right]=\left[S_{j}, t\right]=0$.

The passage from the $t^{j} D_{t}^{j}$-formulation to the $\left(D_{t} t\right)^{j}$-formulation poses no problem, because

$$
t^{\jmath} D_{t}^{j}=\left(D_{t} t\right)^{\jmath}+\sum_{0 \leq k \leq j-1} C_{j k}\left(D_{t} t\right)^{k}, C_{\jmath k} \in \mathbb{Z}
$$

Our equation $Q v=g$ is equivalent to the following integral equation:

$$
v=H\left[g+\sum_{j=0}^{m-1}\left(t^{k, R}+t S_{j}\right)\left(D_{t} t\right)^{\jmath} v\right] .
$$

Here $H$ is the integral operator introduced in $\S 2$. We are going to solve it by successive approximation. Define a sequence of functions $\left\{v_{p}(t, x)\right\}_{p}$ by

$$
\begin{aligned}
v_{0} & =0 \\
v_{p+1} & =H\left[g+\sum_{j=0}^{m-1}\left(t^{k}, R j+t S_{j}\right)\left(D_{t} t\right)^{\prime} v_{p}\right] \text { for } p \geq 0 .
\end{aligned}
$$


The solution $v$ shall be obtained as $v=\lim _{p} v_{p}$. In order to prove convergence, we set $w_{p}=\left(D_{t} t\right)^{m}\left(v_{p+1}-v_{p}\right)$, which satisfies

$$
w_{p+1}=\left(D_{t} t\right)^{m} H\left[\sum_{\jmath=0}^{m-1}\left(t^{k}, R_{\jmath}+t S_{\jmath}\right) G_{m-\jmath} w_{p}\right] \text { for } p \geq 0 .
$$

The limit $v=\lim _{p} v_{p}$ exists if $\sum_{p} w_{p}$ is convergent because $\left(D_{t} t\right)^{m}$ is a topological automorphism.

Since $|g(t, x)| \leq C_{g} / d(x)^{m_{g}}$, a similar estimate holds true for $\imath_{1}^{\prime}=H[g]$. So there exist constants $C^{\prime}>0$ and $a>0$ such that $\left|w_{0}(t, x)\right| \leq C^{\prime} / d(x)^{a}$ holds in a sufficiently small neighborhood of $(t, x)=(0,0)$.

We are going to show that there exists a constant $C>0$ independent of $p$ and $(t, x)$ such that

$$
\left|w_{p}(t, x)\right| \leq C^{p+1} \frac{|t|^{p}}{d(x)^{m p+a}}
$$

for $|t|<\widetilde{T}, x \in \widetilde{\Omega}, p \geq 0$. Here $\widetilde{T}$ is a sufficiently small positive constant and $\widetilde{\Omega}$ is a sufficiently small neighborhood of $x=0$.

The case $p=0$ has already been proved. Assume that (1) is true for $p$. Then by Lemnia 2 we have

$$
\left|G_{m-j} w_{p}(t, x)\right| \leq \frac{C^{p+1}}{(p+1)^{m-j}} \frac{|t|^{p}}{d(x)^{m p+a}} .
$$

Now we need the following

Lemma 4. Let $F(x)$ be a holomorphic function in a domain $U$ of $\mathbb{C}_{x}^{n}$. Denote by' $d(x)$ the distance from $x \in U$ to the boundary of $U$. Assume that $|F(x)| \leq 1 / d(x)^{l}$ for $x \in U$. Then we have $\left|D_{1} F(x)\right| \leq e(1+l) / d(x)^{1+l}, x \in U, j=1,2, \ldots, n$.

Proof. Assume $j=1$. Then

$$
D_{1} F(x)=\frac{1}{2 \pi i} \oint_{\Gamma} \frac{F\left(y, x_{2}, \ldots, x_{n}\right)}{\left(y-x_{1}\right)^{2}} d y
$$

where $\Gamma$ is a circle defined by $\Gamma=\left\{y:\left|y-x_{1}\right|=d(x) /(1+l)\right\}$.

Then for $y \in \Gamma$, we have

$$
d\left(y, x_{2}, \ldots, x_{n}\right) \geq d(x)-\frac{1}{1+l} d(x)=\frac{l}{1+l} d(x) .
$$

The remaining part of the proof, in which the inequality $\{(1+l) / l\}^{l} \leq e$ is used, is routine.

We come back to the proof of (1). Since $t^{k} R_{j}+t S, \in t \mathfrak{D}(m-j)$, where $\mathfrak{D}(m-j)$ is the set of differential operators of order $\leq m-j$, Lemma 4 and (2) 
imply that

$$
\begin{gathered}
\left|\left(t^{k, R}+t S_{\jmath}\right) G_{m-\jmath} w_{p}\right| \\
\leq C^{p+1} C_{j}^{\prime} \frac{(m p+a+1)_{m-1}}{(p+1)^{m-\jmath}} \frac{||^{p+1}}{d(x)^{m p+m+a}}
\end{gathered}
$$

where $C_{j}^{\prime}$ is a positive constant independent of $p$. Here we have used the Pochhammer symbol:

$$
(\lambda)_{n}=\lambda(\lambda+1) \cdots(\lambda+n-1) .
$$

It is easy to see that $(m p+a+1)_{m-\jmath} /(p+1)^{m-j}$ is bounded by a positive constant independent of $p$. Therefore induction proceeds because of Proposition 2 , if $C$ is sufficiently large. The proof of (1) is now complete.

Next we will give a more precise estimate than (1). In its proof, the following lemma will be necessary:

Lemma 5. Let $M$ be a positive constant. Assume that

$$
|w(l, x)| \leq \frac{||^{k}}{d(x)^{l+a}}
$$

holds for $|t|<\widetilde{T}, x \in \widetilde{\Omega}, k>0, l>0$. Moreover suppose that $0<l / k \leq M$. Then there exist positive constants $C_{j}^{(1)}=C_{j}^{(1)}(a, M)$ and $C_{j}^{(2)}=C_{j}^{(2)}(a, M)$ independent of $(k, l)$ and $(t, x)$ such that

$$
\begin{aligned}
\left|t^{k}, R, G_{m-\jmath} w(t, x)\right| & \leq C_{j}^{(1)} \frac{|t|^{k_{j}}}{d(x)^{m-j}} \frac{|t|^{k}}{d(x)^{l+a}} \\
\left|t S, G_{m-j} w(t, x)\right| & \leq C_{\jmath}^{(2)} \frac{1}{k} \frac{|t|}{d(x)^{m-1}} \frac{|t|^{k}}{d(x)^{l+a}}
\end{aligned}
$$

for $|t|<\widetilde{T}, x \in \widetilde{\Omega}$.

Proof. There exists a positive constant $C(R$,$) such that$

$$
\left|R_{\jmath} G_{m-\jmath} w(t, x)\right| \leq C(R,) \frac{(l+a+1)_{m-\jmath}}{(k+1)^{m-\jmath}} \frac{|t|^{k}}{d(x)^{l+a+m-\jmath}} .
$$

If $0<l / k \leq M$, then $(l+a+1)_{m-\jmath} /(k+1)^{m-\jmath}$ is bounded by a positive constant independent of $(k, l)$.

Next, since ord $S_{1} \leq_{m}-j-1$, there exists a positive constant $C\left(S_{j}\right)$ such that 


$$
\begin{aligned}
\left|S_{j} G_{m-j} w(t, x)\right| & \leq C\left(S_{j}\right) \frac{(l+a+1)_{m-j-1}}{(k+1)^{m-j}} \frac{|t|^{k}}{d(x)^{l+a+m-j-1}} \\
& \leq C\left(S_{j}\right) \frac{(l+a+1)_{m-j-1}}{(k+1)^{m-j-1}} \frac{1}{k+1} \frac{|t|^{k}}{d(x)^{l+a+m-j-1}} .
\end{aligned}
$$

If $0<l / k \leq M$, then $(l+a+1)_{m-\jmath-1} /(k+1)^{m-j-1}$ is bounded by a positive constant independent of $(k, l)$.

Fix a positive integer $q \geq 1$ and put

$$
C_{3}=A \max \left\{C_{j}^{(\imath)} ; i=1,2, j=0,1, \ldots, m-1\right\},
$$

where $A$ is as in Proposition 2.

Proposition 3. If $p \geq q$, we have

$$
\left|w_{p}(t, x)\right| \leq C_{3}^{p-q}\left(\sum_{,}^{\prime} \frac{|t|^{k_{j}}}{d(x)^{m-j}}+\frac{m}{q} \frac{|t|}{d(x)^{m-1}}\right)^{p-q} \cdot C^{q} \frac{|t|^{q}}{d(x)^{m q+a}}
$$

for $|t|<\widetilde{T}, x \in \widetilde{\Omega}$. Here $\sum_{j}^{\prime}$ is a sum with respect to the $j$ 's such that $N_{j}(Q) \neq \emptyset$.

Proof. The case $p=q$ is included in (1).

Assume that the estimate holds for $p$. It can be written in the following form:

$$
\left|w_{p}(t, x)\right| \leq \sum_{(k, l) \in I p} C_{k l}^{(p)} \frac{|t|^{k}}{d(x)^{l+a}}
$$

where $I_{p}$ is a finite subset of $\{(k, l) ; k \geq q, l \geq m q, 0<l / k \leq m\}$ and $C_{k l}^{(p)}$ is a positive constant. By using Lemma 5 and Proposition 2, we obtain

$$
\begin{aligned}
& \left|w_{p+1}(t, x)\right| \\
& \leq \sum_{(k, l) \in I_{p}} A C_{k l}^{(p)}\left(\sum_{j}^{\prime} C_{j}^{(1)} \frac{|t|^{k_{j}}}{d(x)^{m-j}}+\sum_{j=0}^{m-1} C_{j}^{(2)} \frac{1}{q} \frac{|t|}{d(x)^{m-1}}\right) \frac{|t|^{k}}{d(x)^{l+a}} \\
& \leq A\left(\sum_{j}^{\prime} C_{j}^{(1)} \frac{|t|^{k_{j}}}{d(x)^{m-j}}+\sum_{j=0}^{m-1} C_{j}^{(2)} \frac{1}{q} \frac{|t|}{d(x)^{m-1}}\right) \sum_{(k, l) \in I_{p}} C_{k l}^{(p)} \frac{|t|^{k}}{d(x)^{l+a}} \\
& \leq C_{3}\left(\sum_{j}^{\prime} \frac{|t|^{k_{j}}}{d(x)^{m-j}}+\frac{m}{q} \frac{|t|}{d(x)^{m-1}}\right) \sum_{(k, l) \in I_{p}} C_{k l}^{(p)} \frac{|t|^{k}}{d(x)^{l+a}} .
\end{aligned}
$$

Hence induction proceeds.

For $q=1,2, \ldots$, set

$$
\widetilde{\Omega}_{q}=\left\{(t, x) ;|t|<\widetilde{C} d(x)^{I(Q)},|t|<\frac{q}{3 m C_{3}} d(x)^{m-1}, x \in \widetilde{\Omega}\right\}, \tilde{C}>0 .
$$

Then we have 
Proposition 4. There exists a positive constant $\widetilde{C}$ independent of $q$ such that the series $\sum_{p \geq 0} w_{p}$ is convergent in $\widetilde{\Omega}_{q}$.

Proof. We have only to prove the convergence of $\sum_{p \geq q} w_{p}$.

First note that $I(Q)=\max \left\{(m-j) / k_{j} ; N_{j}(Q) \neq \emptyset\right\}$. Hence $k_{j} I(Q) \geq m-j$ and $d(x)^{k, I}(Q) \leq d(x)^{m-\jmath}$. Here $\widetilde{\Omega}$ is assumed to be so small that $d(x)<1$ for any $x \in \widetilde{\Omega}$.

Choose $\widetilde{C}>0$ so that $\widetilde{C}^{k_{j}}<1 / 3 m C_{3}$ holds for all $j \in\left\{j ; N_{j}(Q) \neq \emptyset\right\}$. Then if $|t|<\widetilde{C} d(x)^{I(Q)}$, we have

$$
\sum_{j}^{\prime} \frac{|t|^{k_{1}}}{d(x)^{m-j}} \leq \sum_{j}^{\prime}\left(\frac{|t|}{d(x)^{I(Q)}}\right)^{k_{i}} \leq \sum_{j}^{\prime} \widetilde{C}^{k_{1}}<\frac{1}{3 C_{3}} .
$$

Next, if $|t|<q d(x)^{m-1} / 3 m C_{3}$, then

$$
\frac{m}{q} \frac{|t|}{d(x)^{m-1}}<\frac{1}{3 C_{3}}
$$

By using these two estimates and Proposition 3, we obtain

$$
\left|w_{p}\right| \leq\left(\frac{2}{3}\right)^{p-q} C^{q} \frac{\mid t^{q}}{d(x)^{m q+a}}, \quad p \geq q,
$$

for $|t|<\widetilde{T}, x \in \widetilde{\Omega}$. The convergence of $\sum_{p \geq q} u u_{p}$ follows.

Since $q$ is arbitrary and $\widetilde{C}$ and $C_{3}$ are independent of $q$, we conclude that $\sum_{p \geq 0} w_{p}$ is convergent in

$$
\cup_{q \geq 1} \widetilde{\Omega}_{q}=\left\{(t, x) ;|t|<\widetilde{C} d(x)^{I(Q)}, x \in \widetilde{\Omega}\right\} .
$$

The convergence of $\lim _{p} v_{p}$ follows and the proof of Main Theorem in the weight 0 case is now complete, because $I(P)=I(Q)$.

\section{\$4. End of the Proof}

Let us prove Main Theorem in the general case. We look for $u$ in the form

$$
u(t, x)=\sum_{\lambda=0}^{m-m^{\prime}-1} \frac{1}{\lambda !} f_{\lambda}(x) t^{\lambda}+t^{m-m^{\prime}} v(t, x) .
$$

The initial condition is trivially satisfied. The equation $P u=f$ is equivalent to

$$
P t^{m-m^{\prime}} v=f-P\left\{\sum_{\lambda=0}^{m-m^{\prime}-1} \frac{1}{\lambda !} f_{\lambda}(x) t^{\lambda}\right\}
$$

of which the right hand side is a known holomorphic function. It is easy to see 
that $P t^{m-m^{\prime}}$ is a Fuchsian operator of weight 0 and we have $N\left(P t^{m-m^{\prime}}\right)=N(P)$, which implies that $I\left(P t^{m-m^{\prime}}\right)=I(P)$. Moreover we can show that

$$
\begin{aligned}
V & =\left\{x ; C(\lambda, x)=0 \text { for some integer } \lambda \geq m-m^{\prime}\right\} \\
& =\left\{x ; C^{\prime}(\lambda, x)=0 \text { for some integer } \lambda \geq 0\right\} .
\end{aligned}
$$

where $C^{\prime}$ is the characteristic polynomial of $P t^{m-m^{\prime}}$. We have reduced the problem to the weight 0 case, which was dealt with in $\S 3$.

\section{Acknowledgement}

The author thanks the referee for helpful comments.

\section{References}

[1] Yamane, H., Nonlinear Singular First Order Partial Differential Equations Whose Characteristic Exponent Takes a Positive Integral Value, Publ. RIMS, Kyoto Univ., 33 (1997). 801-811.

[2] Baouendi M.S. and Goulaouic G., Cauchy Problems with Characteristıc Initial Hypersurface, Comm. Pure Appl. Math., 26 (1973), 455-475.

[3] Tahara H., Fuchsian type equations and Fuchsian hyperbolıc equations, Japan. J. Math., 5 (1979). 245-347.

[4] Fujıie S.. Singular Cauchy problems of higher order with characteristic surface, J. Math. Kyoto Liniv.., 33 (1993) . 1-27

[5] Ouchı S. Singularitıes of solutions of equations with noninvolutıve characteristıcs- I, the case of second order Fuchsian equations. J. Math. Soc. Japan. 45 (2) (1993), 215-251 